\title{
Basic geometries of the new closed circuit wind tunnel of the Universiti Putra Malaysia
} (UPM)

\begin{abstract}
The existing UPM low speed wind tunnel was usually occupied by students, who carried out their final year projects or postgraduate researches, so that there was hardly free time slot for any additional testing work. Due to this reason, a new wind tunnel project has been started recently. Some basic specifications of the new tunnel have been pre-selected before the project was started, which comprised the following design decisions: a tunnel speed of 50 $\mathrm{m} / \mathrm{s}$, a test section area of $1 \mathrm{xl} \mathrm{m} 2$, and a closed circuit tunnel type. It wouldnâ be difficult to perceive that this pre-selection was made based on some of the trade-off results among the projectôs options and constraints. This paper is aimed to present a simple analysis on the design of the new tunnel, focusing only on its basic geometries. Some design decisions that have been made related to its basic geometries are analyzed and reported in this paper. This analysis may be considered as a design verification of the new tunnel or even perhaps be regarded as scientific justification for its existence.
\end{abstract}

Keyword: Experimental aerodynamics; Flow quality; Subsonic wind tunnel design 